\title{
Research on a New Fiber-Optic Axial Pressure Sensor of Transformer Winding Based on Fiber Bragg Grating
}

\author{
Yuan LIU*, Lianqing LI, Lin ZHAO, Jiqiang WANG, and Tongyu LIU \\ Shandong Provincial Key Laboratory of Optical Fiber Sensing Technologies, Laser Institute of Shandong Academy of \\ Sciences, Jinan, 250014, China \\ *Corresponding author: Yuan LIU E-mail: yuan.liu@sdlaser.cn
}

\begin{abstract}
Based on the principle of the fiber Bragg grating, a new type of fiber-optic pressure sensor for axial force measurement of transformer winding is designed, which is designed with the structure of bending plate beam, the optimization of the packaging process, and material of the sensor. Through the calibration experiment to calibrate the sensor, the field test results of the Taikai transformer factory show that the sensitivity of the sensor is $0.133 \mathrm{pm} / \mathrm{kPa}$ and the repeatability error is $2.7 \% \mathrm{FS}$. The data of the fiber-optic pressure sensor in different positions maintain consistent and repeatable, which can meet the requirement of the real-time monitoring of the axial force of transformer winding.
\end{abstract}

Keywords: Transformer winding; pressure sensor; fiber Bragg grating (FBG); axial force

Citation: Yuan LIU, Lianqing LI, Lin ZHAO, Jiqiang WANG, and Tongyu LIU, "Research on a New Fiber-Optic Axial Pressure Sensor of Transformer Winding Based on Fiber Bragg Grating," Photonic Sensors, 2017, 7(4): 365-371.

\section{Introduction}

It is important to detect the mechanical condition and the change trend of the transformer winding. When the transformer short-circuit occurs, due to the generated leakage magnetic field, the winding will produce a greater force. The axial electro-dynamic force is generated internally to the winding by the radial component of the leakage magnetic flux. It can make the coil outward expansion, even causing the winding deformation fault $[1,2]$. Nowadays, the major detection methods for the winding condition include the short-circuit impedance method and the frequency response analysis (FRA) method. These two methods require high precision and anti-interference, so the systems are more complex. If the performances are not good enough, the monitoring results will produce a greater error. The optical fiber technology has many advantages, such as anti-interference ability, high precision, good repeatability, and simple installation, especially for the transformer winding dynamic axial force on-line monitoring [3]. At present, there are many domestic and international studies on the pressure sensitizing structure, packaging materials and technology, and temperature compensation methods of pressure sensors based on fiber Bragg grating (FBG) [4, 5]. The common FBG-based pressure sensor can be divided into the polymer encapsulation, planar diaphragm packaging, and other different sensitization structures according to different package types and structures [6]. For general polymer packaging, the temperature and pressure cross sensitivity is not suitable for high temperature measurement although the pressure is sensitive. For the planar diaphragm packaging, the 
small changes in the diaphragm easily lead to uneven pressure to generated signal distortion. In addition, in order to meet the requirements of dynamic measurement, the dynamic response of the winding is required to satisfy the requirements of the instantaneous strain recovery of the packaging structure and material [7]. In this paper, according to the characteristic of winding axial force, a new type of FBG-based pressure sensor based on the bending beam sensitivity structure is designed, and the reference fiber grating method effectively solves the problem of temperature cross sensitivity, and the experiment and field test are carried out.

\section{Fiber-optic sensoring principles}

\subsection{FBG sensoring principle}

FBG-based sensor works like a wavelength selector reflector, the incident light [wavelength $\left(\lambda_{B}\right)$ ] satisfying the Bragg diffraction condition is reflected at FBG, and the other wavelengths pass through all without being affected [8]. The reflection spectrum peaks at the FBG central wavelength $\lambda_{B}$ is given as

$$
\lambda_{B}=2 n_{\text {eff }} \times \Lambda
$$

where $\lambda_{B}$ is the central wavelength of the reflected light, $n_{\text {eff }}$ is the effective refractive index, and $\Lambda$ is the pitch.

The wavelength will change by the temperature and strain. The FBG based on the co-production of temperature and strain can be expressed as

$$
\Delta \lambda / \lambda_{B}=\left(1-P_{e}\right) E+(\alpha+\zeta) \Delta T
$$

where $\Delta \lambda$ is the change in wavelength, $P_{e}$ is the photo elastic coefficient, $E$ is the axial force, $\Delta T$ is the temperature change, $\alpha$ is the thermal expansion coefficient of optical fiber, and $\zeta$ is the thermo-optical coefficient of optical fiber.

In order to solve the influence of temperature on the pressure measurement, the reference grating method is used to eliminate the temperature effect, so the strain can be expressed as

$$
\varepsilon=\left(\Delta \lambda_{B 1} / \Delta \lambda_{B 1}-\Delta \lambda_{B 2} / \Delta \lambda_{B 2}\right) / K_{\varepsilon}
$$

where $\lambda_{B 1}$ is the wavelength of FBG1, $\lambda_{B 2}$ is the wavelength of $\mathrm{FBG} 2$, and $K_{\varepsilon}$ is the pressure sensitivity coefficient. According to (3), the pressure can be obtained without the influence of temperature.

\subsection{Sensitivity structure design principle}

There are beam, spoke, column, pressure ring, and other structures of pressure sensors with its elastomerric structures. Each has its own characteristics and advantages and is applied in different occasions. In this paper, we use the bending plate structure to make up the fiber-optic pressure sensor $[9,10]$. In Fig. 1, the distributed load can be decomposed into a set of $2 N+1$ smaller concentrated forces $W(x i)$ as follows:

$$
W=\sum_{2 N+1}^{1} W(x i)
$$

In order to achieve better dynamic sensitivity, we should get the maximum deflection of sensor beam, and the calculation formula is given as

$$
\begin{gathered}
f_{0, l / 2}=W_{i} x_{i}\left(3 l^{2}-4 x_{i}^{2}\right)\left(4 E b h^{3}\right)^{-1} \\
0 \leq x_{i} \leq l / 2 \\
f_{l / 2, l}=W_{i}\left(l-x_{i}\right)\left(3 l^{2}-4\left(l-x_{i}\right)^{2}\right)\left(4 E b h^{3}\right)^{-1} \\
l / 2 \leq x_{i} \leq l
\end{gathered}
$$

where $E$ is the elastic modulus of beam, $l$ is the distance of pressure from the fulcrum, $b$ is the beam width, $h$ is the beam height, and $x$ is the left fulcrum distance.

According to (5) and (6), when $x_{i}=l / 2$, the deflection has the maximum value which is in the middle of the beam, and the formula is given as follows:

$$
f_{\max }=W l^{3}\left(4 E b h^{3}\right)^{-1} .
$$

In the case of other conditions unchanged, the maximum deflection $f$ in the middle of the beam approaches the force $W$. The sensitivity $K$ is expressed as follows:

$$
K=(\Delta \lambda / \lambda)_{\max } / W .
$$

The sensor is designed by this structure of square bending plate beam. Three-dimensional stress simulation of the sensor is shown in Fig. 1. 

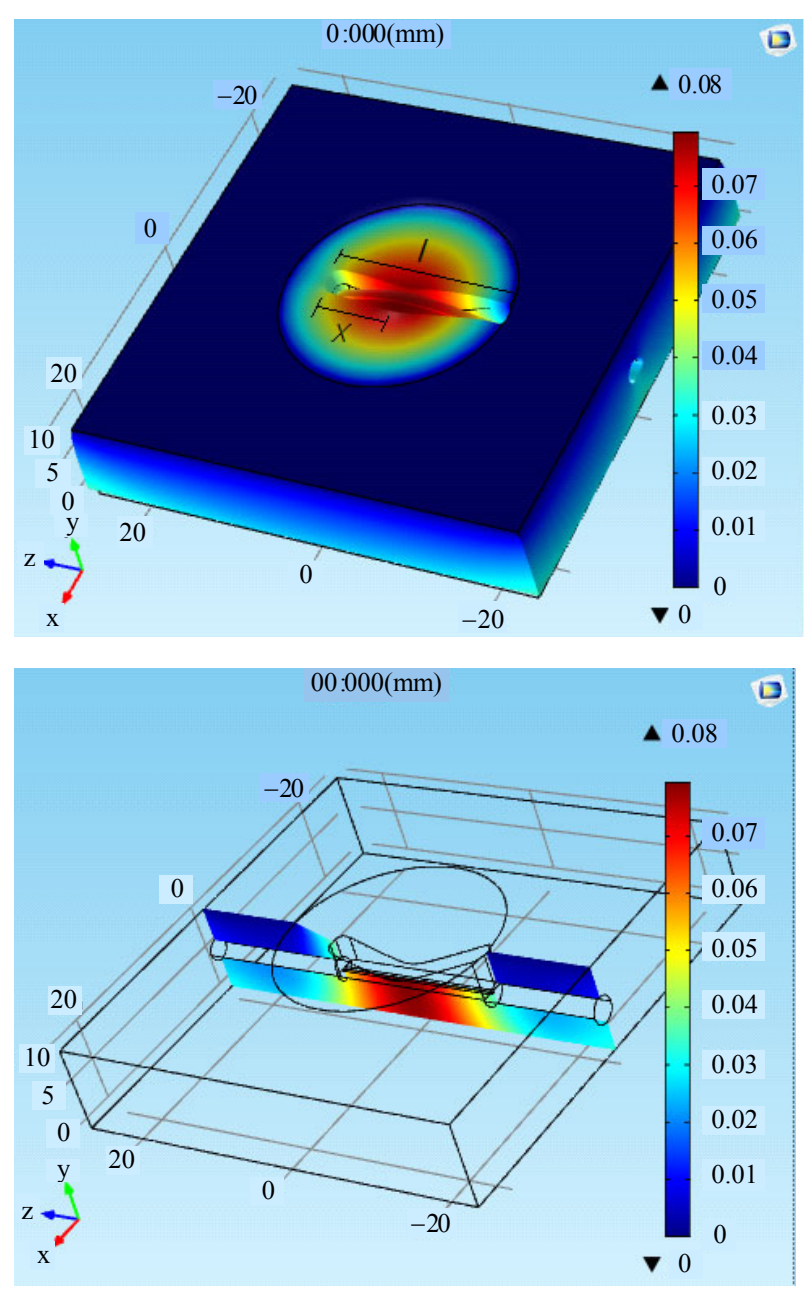

Fig. 1 Three-dimensional stress simulation of fiber-optic sensor.

According to the analysis, when a load is applied to the upper part of the sensor, the sensor pressures are concentrated on the middle of the bow. The pressure FBG is fixed in it. Further optimization of the design of its structure is to make the wavelength change of the FBG sensor in full-scale greater than $1 \mathrm{~nm}$.

\section{Sensor package and sensoring system}

\subsection{Sensor package}

The fiber-optic pressure sensor is used to monitor the transformer winding axial force, because the material of the sensor has good insulation, mechanical property, and high temperature resistance. We select polyether ether Ketone (PEEK) special engineering plastics as sensor's substrate material. PEEK resin has advantages of large rigidity, better dimensional stability, and very small linear expansion coefficient, and it is close to the metal aluminum material which can be used at $250{ }^{\circ} \mathrm{C}$ for a long period. The fiber-optic pressure sensor is shown in Fig. 2.

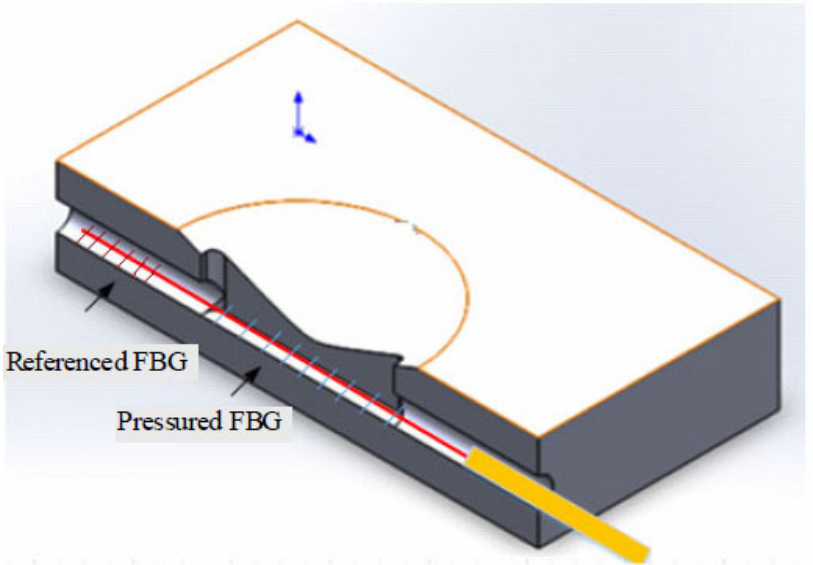

(a)

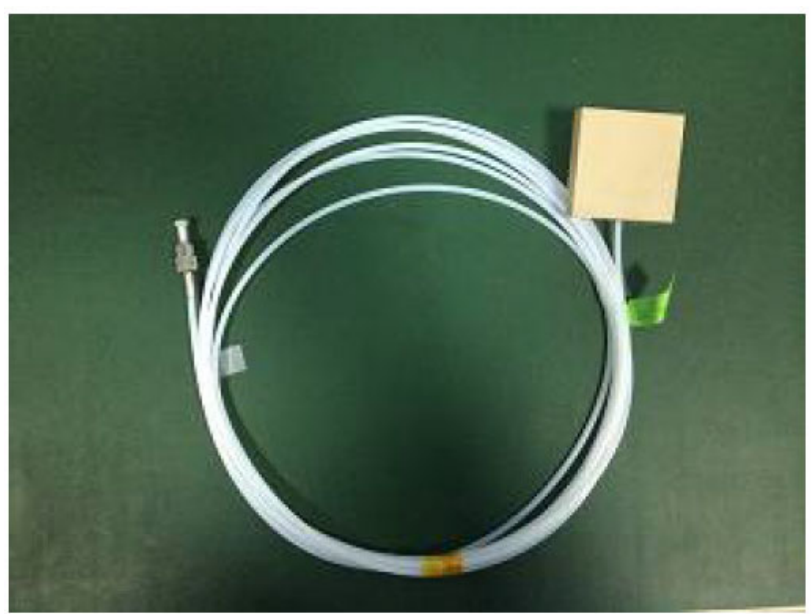

(b)

Fig. 2 Structure drawing and photo of the fiber-optic pressure sensor: (a) fiber-optic pressure sensor for package and (b) photograph of the fiber-optic pressure sensor.

In Fig. 2(a), it shows that there are two FBGs, namely the pressured FBG1 and the referenced FBG2, which are enclosed in the groove. The FBG1, which measures the axial pressure, is located in the arcuate groove which is in the center of the sensor and is pasted on the bottom of the groove by glue. The FBG2, which measures the temperature, is in a non-stressed state. When the upper plane or the lower plane of the fiber-optic pressure sensor is in force, the arcuate groove is subjected to force, which can cause the change in the wavelength of the FBG1. 
So the pressure which eliminates temperature effect can be given by FBG1 and FBG2.

\subsection{Sensoring calibration and test experiment}

Light is emitted by the laser in the FBG demodulator via coupler and fiber to the fiber-optic pressure sensor and then is reflected by the FBG in the sensor, and then, via optical fiber, coupler to demodulation module which converts the optical signal to electrical signal $[11,12]$. The demodulator performance indicators are: demodulation wavelength range: $1522 \mathrm{~nm}-1567 \mathrm{~nm}$, wavelength resolution: $0.1 \mathrm{pm}$, and data sampling frequency: $8 \mathrm{kHz}$. The relationship between the change in FBG wavelength, and the applied load can be obtained by applying different loads to the fiber-optic pressure sensor.
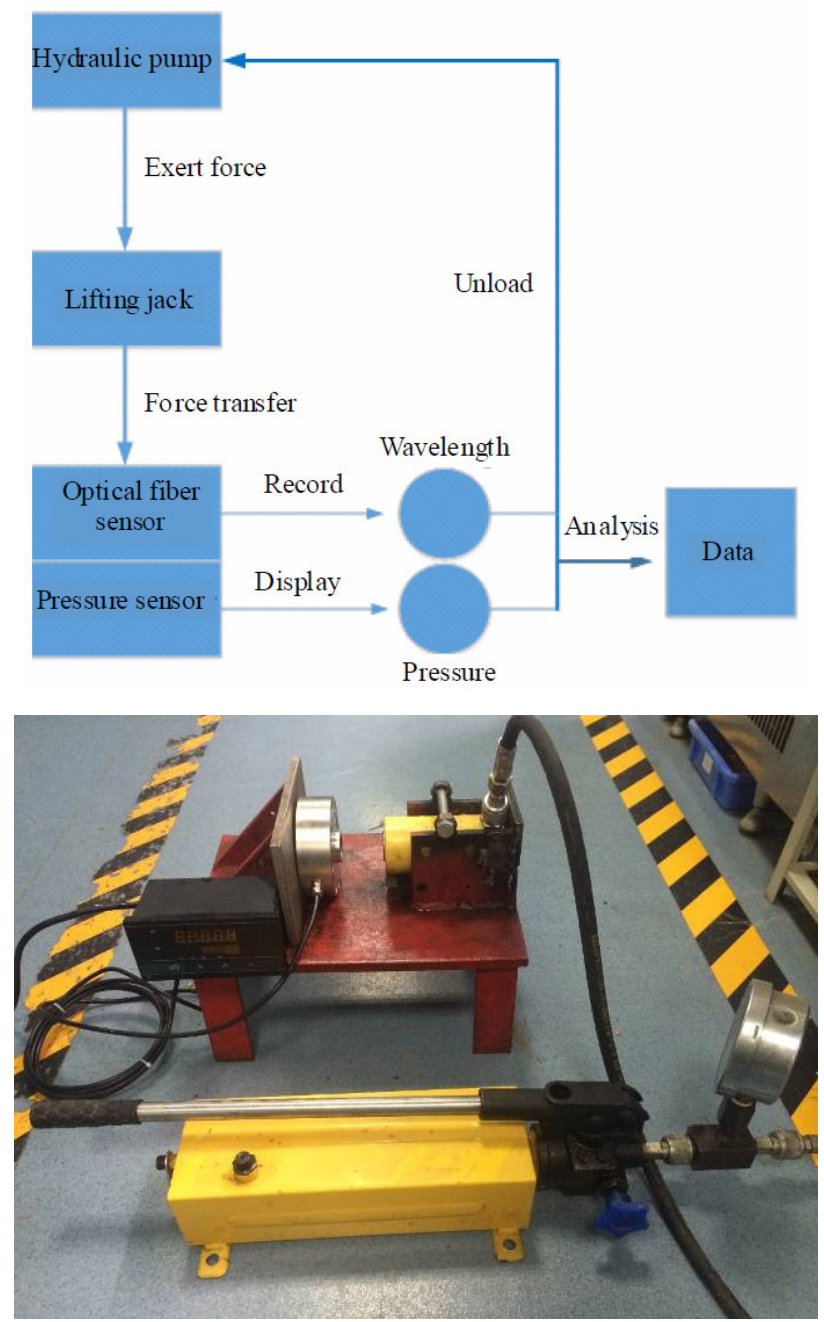

Fig. 3 Pressure sensor test system flow chart and picture.
To establish the calibration platform of FBG pressure sensor, the platform picture is shown in Fig. 3. The equipment is constituted of manual pressure pump, jack, structure platform, and high-precision pressure sensor (US transcell DBSL-XS-10T, its range is $0 \mathrm{~T}-10 \mathrm{~T}$, accuracy: $0.05 \% \mathrm{FS}$ ).

When the pressure sensor is calibrating, a high precision pressure sensor is installed in the middle of the jack. The load points corresponding to $0 \mathrm{MPa}$, $2 \mathrm{MPa}, 4 \mathrm{MPa}, 6 \mathrm{MPa}, 8 \mathrm{MPa}, 10 \mathrm{MPa}, 12 \mathrm{MPa}$, and $14 \mathrm{MPa}$ are pressured by the manual hydraulic pump. The sensor wavelength value and the pressure value of the high precision pressure sensor are recorded, and the corresponding relationship model is established as shown in Fig. 4. We add the temperature disturbance in this experiment, and the wavelength shift caused by the temperature change of the reference grating FBG2 is eliminated on the pressure grating FBG1 data.

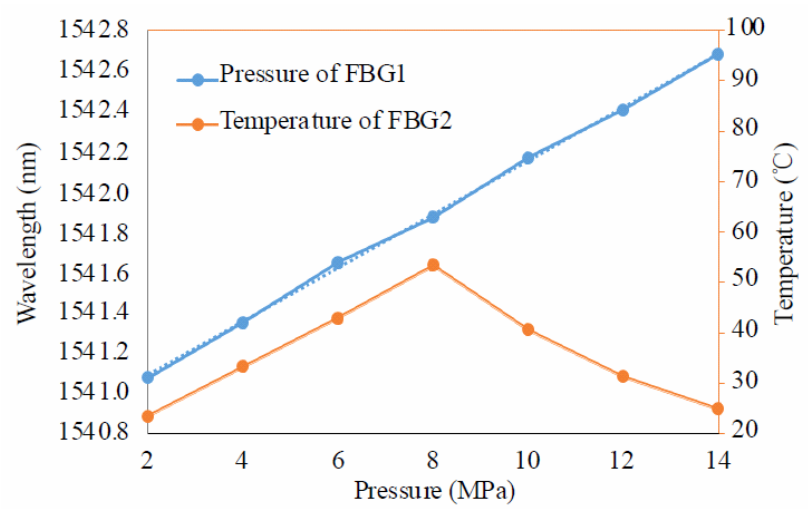

Fig. 4 Pressure sensor calibration curve.

The linear relation between the pressure and wavelength is expressed as follows:

$$
\begin{gathered}
y=0.133 x+1540.812 \\
R^{2}=0.999 .
\end{gathered}
$$

It can be seen that the linear correlation coefficient of the sensor is 0.999 , and the sensitivity of the sensor is $0.133 \mathrm{pm} / \mathrm{kPa}$.

\section{Field test and result in transformer winding}

\subsection{Sensor installation}

The winding axial force field test is carried out 
by installing the fiber-optic pressure sensor inside the transformer winding in Taikai transformer factory. Two fiber-optic pressure sensors are respectively installed on the upper and the lower pressing plates of the transformer winding. The position of the lower sensor is corresponding to the upper sensor, and the other sensor is installed in the middle part. The installation diagram is shown in Fig. 5. When installing, the appropriate insulation gasket is selected for the pressure sensor to install.

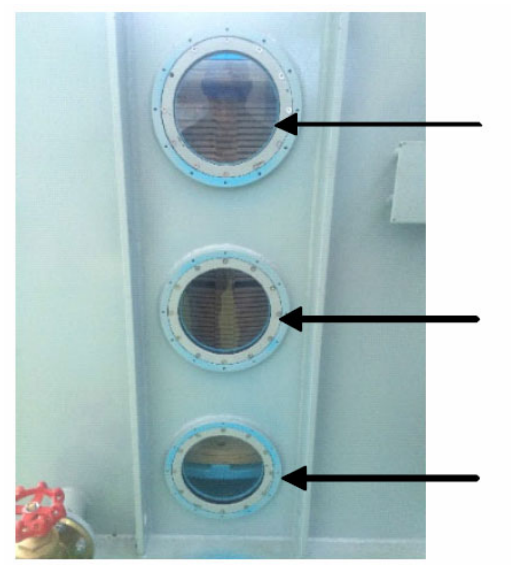

Fig. 5 FBG-based pressure sensor installation diagram.
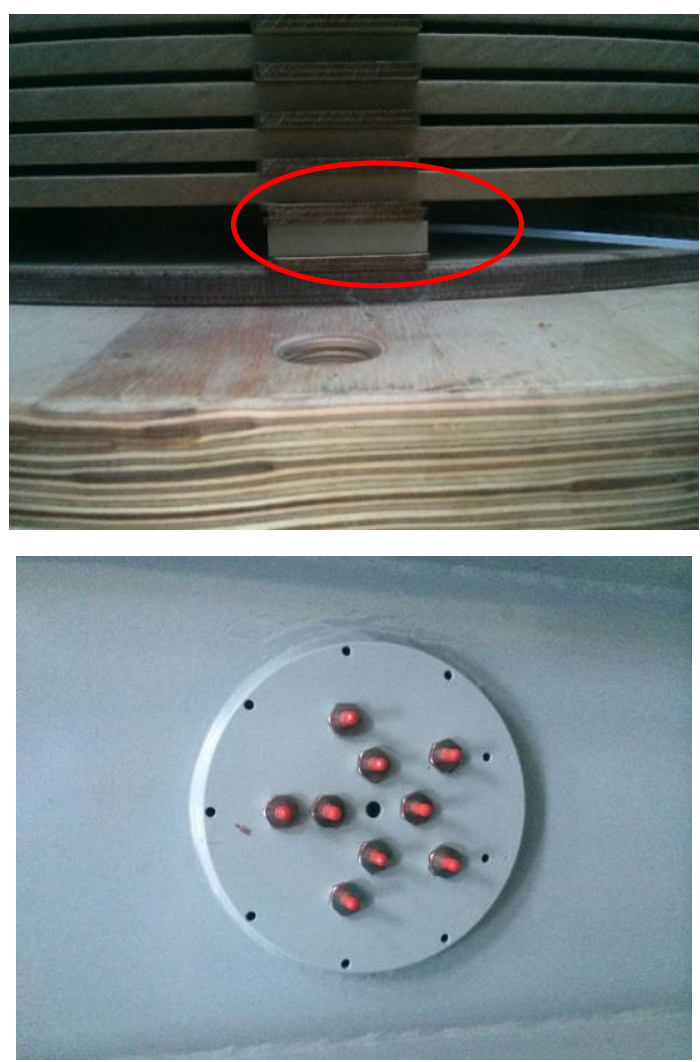

Fig. 6 Fiber-optic pressure sensor installation location and transfixion device.
The sensor is connected to the external optical fiber cable with the transformer transfixion device. The bottom of the transfixion sealed chamber is opened some holes, and the external fiber cable is connected to the demodulator with the metal hose (no more than $6 \mathrm{~km}$ ). The fiber-optic dynamic pressure sensors are placed in the insulation gasket. Figure 6 shows the fiber-optic pressure sensor installation location and transfixion device. Figure 7 shows the field pressure test system of transformer winding.
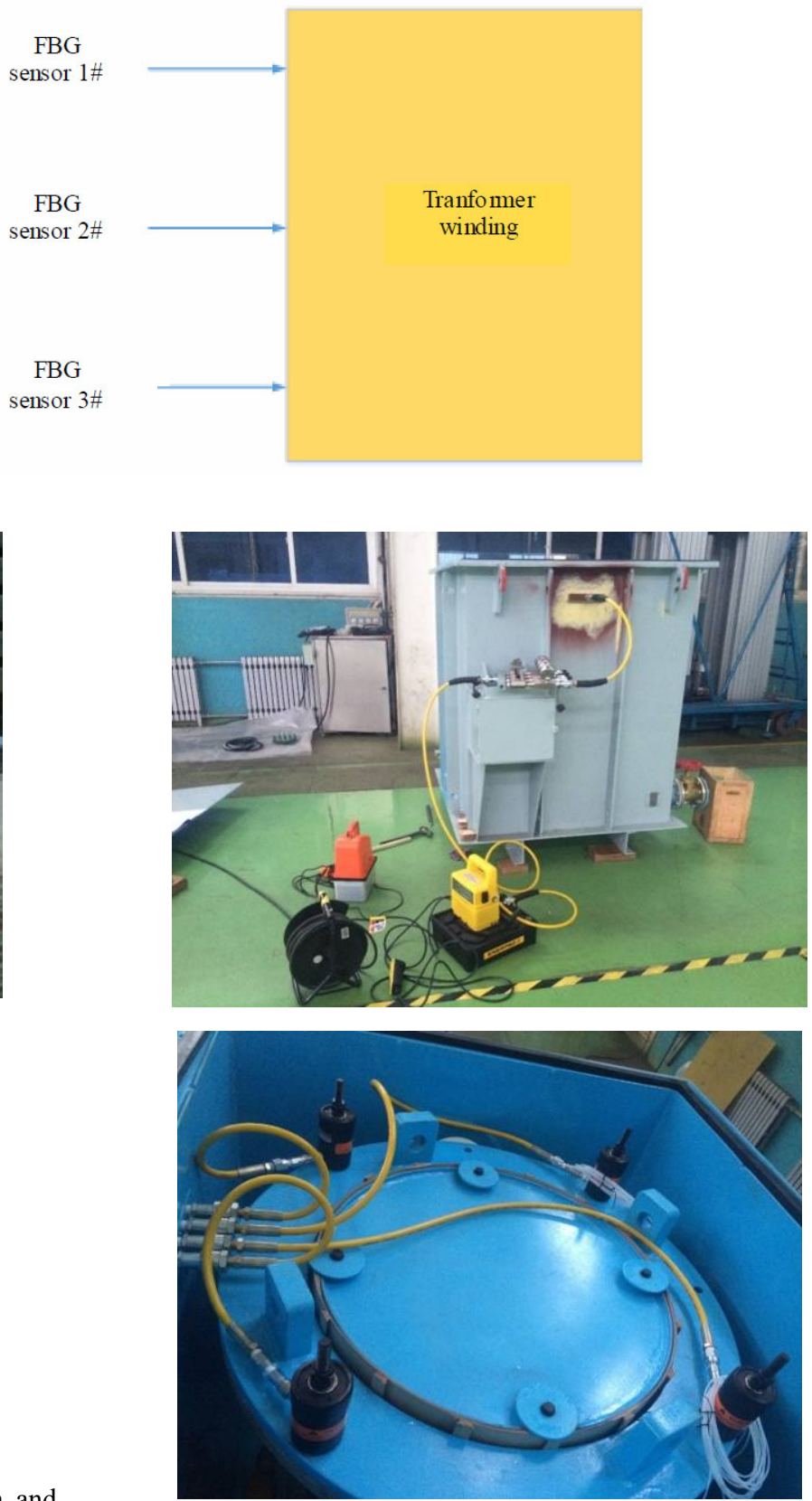

Fig. 7 Field pressure test system of transformer winding. 


\subsection{Results analysis}

Figure 8 shows that each $5 \mathrm{MPa}$ is used as a test point, and a total of 5 test points are obtained. The data curve shows that the data of the fiber optic pressure sensors installed in the upper, middle and lower parts are the same basically. When the maximum pressure is applied, the error of the three sensors is larger.

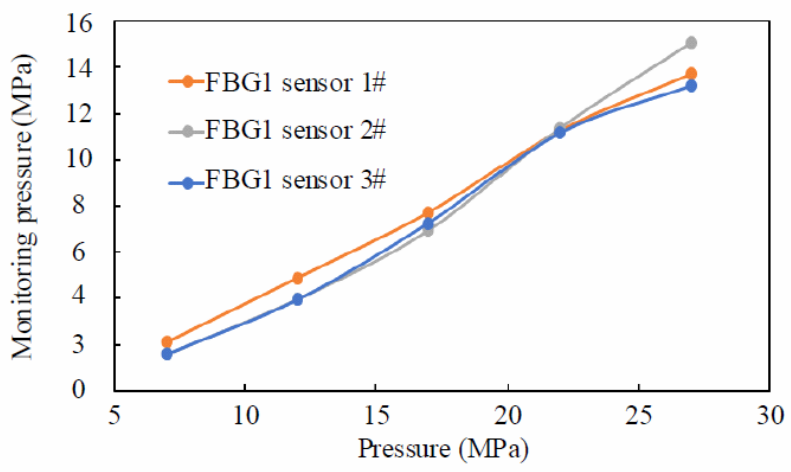

Fig. 8 Field test curve of fiber-optic pressure sensor.

Table 1 shows the data of positive (order from small to large) and negative (order from large to small) stroke calibrations of the sensor. So that the repeatability error can be calculated as

$$
\xi_{s}=3 S / y_{F \cdot S} \times 100 \%
$$

where $S$ is the standard deviation of the sensors, and $y_{F \cdot S}$ is the full scale output value for the pressure sensor. According the calculation, the repeatability error is $S=2.7 \%$.

Table 1 Positive and negative stroke error data.

\begin{tabular}{cccc}
\hline Pressure $(\mathrm{MPa})$ & Positive stroke $(\mathrm{nm})$ & Negative stroke $(\mathrm{nm})$ & Error $(\mathrm{nm})$ \\
\hline 0 & 1540.819 & 1540.819 & 0.000 \\
2 & 1541.079 & 1541.077 & 0.002 \\
4 & 1541.343 & 1541.340 & 0.003 \\
6 & 1541.611 & 1541.608 & 0.003 \\
8 & 1541.873 & 1541.868 & 0.005 \\
10 & 1542.149 & 1542.145 & 0.004 \\
12 & 1542.406 & 1542.404 & 0.002 \\
14 & 1542.682 & 1542.682 & 0.000 \\
\hline
\end{tabular}

The dynamic measurement of the pressure sensor is carried out in order to investigate the dynamic sensing ability. The force rate can be changed by the pressure testing machine, and the sensor detection results are shown in Fig. 9. It can be seen that the sensing sensitivity and linearity of the sensor are not affected by different control rates.

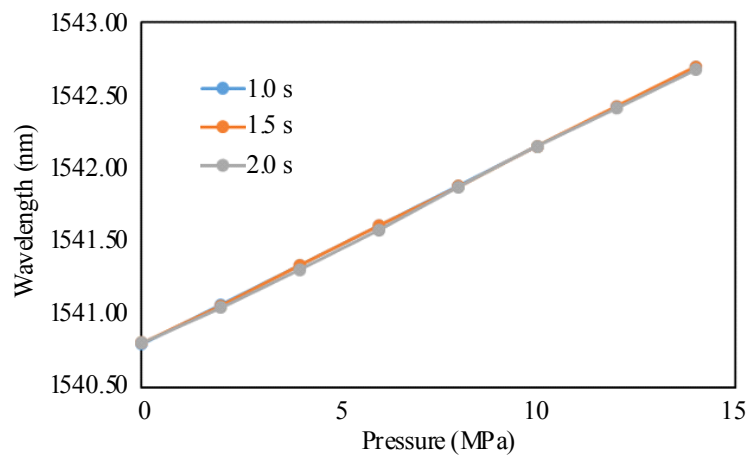

Fig. 9 Test results under different force control rates.

\section{Conclusions}

According to the measurement of axial pressure of transformer winding, a new kind of FBG-based axial pressure sensor based on the design of bending plate beam is designed. The sensitivity of the sensor is $0.133 \mathrm{pm} / \mathrm{kPa}$, and the repeatability error is $2.7 \%$ FS. The transformer winding field experiment shows that the installation of the FBG-based pressure sensor is more convenient and anti-interference, and the field data of the sensor in different positions are basically consistent and repeatable, which can meet the need of the measurement of the axial pressure of the winding. In the future, the packaging materials of the FBG-based pressure sensor can be optimized to reduce the anisotropy affection between the winding insulation gasket and the sensor material. To measure a large amount of data to establish the analysis model of transformer winding axial force in different positions, further simulation analysis is carried out to provide a reliable data model for the pressure monitoring of the transformer winding in operation.

\section{Acknowledgment}

This work was partly supported by the Natural Science Fund Plan of Shandong Province (No. 2016ZRC01104) and Natural Science Foundation Doctoral Fund of Shandong Province (No. ZR2016FB03).

Open Access This article is distributed under the terms of the Creative Commons Attribution 4.0 International License (http://creativecommons.org/ licenses/by/4.0/), 
which permits unrestricted use, distribution, and reproduction in any medium, provided you give appropriate credit to the original author(s) and the source, provide a link to the Creative Commons license, and indicate if changes were made.

\section{References}

[1] W. D. S. Fonseca, D. D. S. Lima, A. K. F. Lima, N. S. Soeiro, and M. V. A. Nunes, "Analysis of electromagnetic-mechanical stresses on the winding of a transformer under inrush currents conditions," International Journal of Applied Electromagnetics and Mechanics, 2016, 50(4): 511-512.

[2] A. Marinescu, G. Opran, M. Teodorescu, I. Dinu, and L. Tascau, "Fibre optic based clamping force monitoring system for power transformers," in Proceeding of 13th International Conference on Optimization of Electrical and Electronic Equipment, Brasov, Romania, 2012, pp. 282-283.

[3] Z. F. Chen, C. H. Zhou, L. Y. Xu, W. Liu, and Z. B. $\mathrm{Xu}$, "On-line monitoring method of transformer winding deformation based on optical fiber stress sensing technology," Electric Age, 2014, 8: 74-76.

[4]Q. Z. Wen, J. H. Zhu, G. N. Li, J. B. Huang, and C. Yu, "The experimental study and structural design of fiber grating pressure sensor for pressure sensitivity enhancement," Journal of Wuhan University, 2012, 58(5): 411-413.

[5] J. Huang, "Development and application of fiber Bragg grating pressure sensors," M.S. dissertation,
Wuhan University of Technology, Wuhan, China, 2013.

[6] A. Sun, X. G. Qiao, Z. A. Jia, T. Guo, and C. Y. Chen, "Strain response of a special cantilever-based fibre Bragg grating," Journal of Optoelectronics Laser, 2004, 15(2): 153-154.

[7] T. Guo, Q. D. Zhao, L. H. Liu, G. L. Huang, L. F. Xue, G. Y. Li, et al., "Light intensity-referred and temperature-insensitive fiber Bragg grating dynamic pressure sensor," Acta Optica Sinica, 2007, 27(2): 208-209.

[8] J. Y. Wang, L. Jiang, Z. R. Sun, B. X. Sun, F. X. Hu, G. D. Zhang, et al., "Research on the surface subsidence monitoring technology based on fiber Bragg grating sensing," Photonic Sensors, 2016, 7(1): 20-21.

[9] H. Ahmad, S. W. Harun, W. Y. Chong, M. Z. Zulkifli, M. M. M. Thant, Z. Yusof, et al., "High-sensitivity pressure sensor using a polymer embedded FBG," Microwave \& Optical Technology Letters, 2008, 50(1): 60-61.

[10] Y. Wang, T. G. Liu, L. N. Liu, and J. F. Jiang, "Study on fiber Bragg grating sensor encapsulated by the alloyed steel," Optical Technique, 2006, 32(6): 923-924.

[11] C. C. Hu, D. S. Zhang, C. Y. Wen, and W. He, "Researches on fiber Bragg grating pressure sensor," Journal of Wuhan University of Technology, 2007, 29(1): 52-54.

[12] Y. Zhao, Q. Y. Meng, and K. Chen, "Novel current measurement method based on fiber Bragg grating sensor technology," Sensors and Actuators, 2006, 126(1): 112-116. 